GRAVITATIONAL RADIATION Magnetic Correlation?

from a Correspondent

THE chief evidence for associating the signals recorded by Weber's gravitational wave detectors with an astronomical source outside the solar system has been the 12-hour periodicity displayed in published plots of event rate as a function of sidereal time. Now a wider analysis of the times of some of Weber's events has been made, and this indicates that there may also be correlations with disturbances of the Earth's magnetic field and with sunspot activity. Such correlations would suggest that Weber's detectors may be influenced by phenomena unconnected with gravitational radiation and may help to resolve some of the problems raised by Weber's observations.

The new analysis has been made by Tyson, Maclennan and Lanzerotti of Bell Laboratories (Phys. Rev. Lett., 30, $1006 ; 1973)$, and follows earlier work by Adamyants, Alekseev and Kososnitsyn in the Soviet Union. The Soviet physicists based their analysis on published times of seventeen coincident events recorded by Weber's detectors at Maryland and Chicago in late 1968 and early 1969. They found a correlation between changes of terrestrial magnetic activity and the days when Weber events were recorded and they also found a rather stronger correlation, involving a time lag of 2 days, between sunspot activity and Weber pulses. These results have generally been regardied as interesting, but of limited significance because of the small number of events analysed.

The group at Bell Laboratories was provided by Weber with data on 262 events recorded with his detector in a period of 121 days beginning in August 1969. They have searched for correlations between the daily rates of Weber's events and many other phenomena including geomagnetic activity, sunspot number, averaged earthquake amplitudes at a point between Maryland and Chicago, tidal strain in the Earth and changes in barometric pressure and in temperature. Correlations at levels above 2 standard deviations are found between the Weber data and geomagnetic, sunspot and earthquake activity; with a time lag of 8 days in the sunspot correlation. The strongest geophysical correlation found was with magnetospheric ring-current intensity and was at the level of 2.7 standard deviations. The rate of occurrence of the gravitational wave events throughout the sidereal day was also investigated and a distribution similar to those reported by Weber was obtained, with peaks occurring twice a day at the times when the detectors would be expected to have maximum sensitivity for gravitational radiation coming from the direction of the galactic centre. This correlation was at the level of 3 standard deviations.

What do these correlations imply? In the case of the magnetospheric correlations the Bell Laboratories group points out that the enhanced low frequency magnetic disturbances which are present at times of high geomagnetic activity may couple into Weber's detectors and produce some "events"; it is known that fluctuations in the magnetic fields can be correlated sufficiently closely in time over distances equal to that from Maryland to Chicago to be recorded as coincident in Weber's equipment. Weber has carried out experiments to search for magnetic coupling to his detectors, but it seems that his published tests were made with magnetic sensing equipment tuned to frequencies related to the resonant frequencies of his detectors. It may be that very low frequency magnetic fluctuations would not be efficiently detected in such tests and might penetrate the steel vacuum tanks and cause vibrations in ferromagnetic materials coupled to the aluminium bars.

It is more difficult to understand the reported sunspot and earthquake correlations, and the Bell Laboratories group does not suggest interpretations here. In fact they do not claim that their analysis indicates the prime source of Weber's events. The point they make is that, as the correlation with some geomagnetic phenomena is almost as strong as the correlation with sidereal time, at least a certain fraction of
Weber's events may be caused by processes other than gravitational radiation.

As the authors indicate, however, the data used in this study represent only a small part of the body of data now collected by Weber and a more extensive analysis might give different results. Clarification of the problem may come from some of the independent experiments with gravitational wave detectors now in progress. None of the results reported to date has confirmed Weber's observations, but most of the experiments have only collected data over relatively short periods, and it may be some time before unambiguous conclusions can be drawn.

\section{MARS}

\section{Are Ice Ages Temporary?}

by our Cosmology Correspondent

At a special seminar held at the University of Sussex on June 8, Professor Carl Sagan, of Cornell University, presented some of the evidence from the Mariner 9 photography which has led him to suggest that Mars may be in a temporary ice age at present, and that more clement conditions recur on the planet periodically. This idea has exciting implications for the prospect of finding evidence of life on the planet.

Sagan's discourse was divided into two parts; the first covered the implications of the great dust storm which was raging when Mariner 9 arrived at Mars, and which seems to be a typical pheno-

\title{
Spiral Structure and Nuclear Activity in Galaxies
}

THE density wave theory can explain many features of the spiral structure of galaxies, but requires a regular input of energy. One recent suggestion is that regeneration (about every $10^{9} \mathrm{yr}$ ) is associated with gas expelled from the galaxy's nucleus. That idea has been followed up by van der Kruit, who describes in Nature Physical Science next Monday (June 25) a model in which a continuous cycle links nuclear activity with regeneration of spiral structure, while angular momentum transport by density waves adds to the production of nuclear energy.

Observational data which help in the development of this model have been carried out at Westerbork by van der Kruit, and reveal two important correlations. First, the relative velocity bewteen gas and the spiral pattern, $\omega_{\perp 0}$, is larger when the ratio of radius at which the rotation curve is maximum $\left(R_{\mathrm{m}}\right)$ to optical radius $\left(R_{\mathrm{o}}\right)$ is smaller. Moreover, a similar relation holds for the compression strength and this ratio; both relations are distance independent.

The Westerbork survey data provide measurements of compression strength for only fourteen galaxies, but use of the $R_{\mathrm{m}} / R_{\mathrm{o}}$ relations and of van den Bergh luminosities enables van der Kruit to extend this sample to twenty-six galaxies with well observed rotation curves. Galaxies with low $R_{n 1} / R_{0}$, and thus strong compression, are relatively more luminous because of enhanced star formation.

Further observational data suggest that galaxies with low $R_{\mathrm{m}} / R_{\mathrm{o}}$ also contain better developed nuclei, showing clear signs of activity. Because nuclear explosions can regenerate the density waves by gas expulsion, but these same density waves then transport angular momentum such that gas and rotational energy are fed into the nucleus, van der Kruit suggests that the observations indicate the presence of a closed cycle, both directions of which operate more strongly in galaxies with low $R_{\mathrm{m}} / R_{\mathrm{o}}$. There is, of course, some scatter in the observations, and the precise nature of the expulsive mechanism remains unknown. This work points the way to a new and interesting approach to the problem of spiral structure, rather than presenting all the answers. 